\title{
Identification of Low-Dimensional Energy Containing / Flux Transporting Eddy Motion in the Atmospheric Surface Layer Using Wavelet Thresholding Methods
}

\author{
GABRIEL KATUL \\ School of the Environment, Duke University, Durham, North Carolina \\ BRANI VIDAKOVIC \\ Department of Statistics, Duke University, Durham, North Carolina
}

(Manuscript received 1 November 1996, in final form 9 June 1997)

\begin{abstract}
The partitioning of turbulent perturbations into a "low-dimensional" active part responsible for much of the turbulent energy and fluxes and a "high-dimensional" passive part that contributes little to turbulent energy and transport dynamics is investigated using atmospheric surface-layer (ASL) measurements. It is shown that such a partitioning scheme can be achieved by transforming the ASL measurements into a domain that concentrates the low-dimensional part into few coefficients and thus permits a global threshold of the remaining coefficients. In this transformation-thresholding approach, Fourier rank reduction and orthonormal wavelet and wavelet packet methods are considered. The efficiencies of these three thresholding methods to extract the events responsible for much of the heat and momentum turbulent fluxes are compared for a wide range of atmospheric stability conditions. The intercomparisons are performed in four ways: (i) compression ratios, (ii) energy conservation, (iii) turbulent flux conservation, and (iv) finescale filtering via departures from Kolmogorov's K41 power laws. For orthonormal wavelet and wavelet packets analysis, wavelet functions with varying time-frequency localization properties are also considered. The study showed that wavelet and wavelet packet Lorentz thresholding can achieve high compression ratios (98\%) with minimal loss in energy (3\% loss) and fluxes (4\%). However, these compression ratios and energy and flux conservation measures are comparable to the linear Fourier rank reduction method if a Lorentz threshold function is applied to the latter. Finally, it is demonstrated that orthonormal wavelet and wavelet packets thresholding are insensitive to the analyzing wavelet.
\end{abstract}

\section{Introduction}

The recognition that wavelet transforms possess mathematical properties capable of quantifying spacescale relations in turbulent flows stimulated much research in fluid dynamics, boundary layer meteorology, and surface hydrology. In particular, the use of wavelet transforms in conjunction with recent compression algorithms suggest a potential reduction in the large degrees of freedom necessary to describe turbulence measurements and simulations (Wickerhauser et al. 1994; Farge et al. 1992; Zubair et al. 1992). Thus far, the combination of wavelets and compression algorithms was used by several investigators with excellent reported reduction in degrees of freedom in describing dynamical features of turbulence. These investigations are based on the premise that turbulence can be decomposed into a dynamical "low-dimensional" active part

Corresponding author address: Dr. Gabriel G. Katul, School of the Environment, Duke University, Box 90328, Durham, NC 277080328.

E-mail: gaby@duke.edu and a "high-dimensional" passive part. Farge et al.'s (1992) simulations demonstrated that the dynamically active part is associated with coherent structures, while the passive part is associated with weak vorticity filaments contributing little to the enstrophy.

In atmospheric surface layer (ASL) experiments, the vorticity field is typically not measured. However, the influence of coherent eddy motion on velocity and temperature time series measurements is well documented (e.g., Qui et al. 1995; Katul et al. 1994a,b; Hayashi 1994; Lu and Fitzjarald 1994; Mahrt and Howell 1994; Gamage and Hagelberg 1993; Collineau and Brunet 1993; Gao and Li 1993; Raupach et al. 1991; and others). In particular, it is recognized that few such organized events contribute significantly to measured variances and turbulent fluxes. Therefore, it is possible to decompose such turbulence measurements into a lowdimensional (active) part responsible for much of the turbulent energy content and heat/momentum fluxes and a high-dimensional passive part that is inertial in the sense of Kolmogorov (1941). Such a decomposition provides a practical approach for (i) identifying coherent eddy motion from velocity and temperature time series measurements, (ii) investigating the potential reduction 

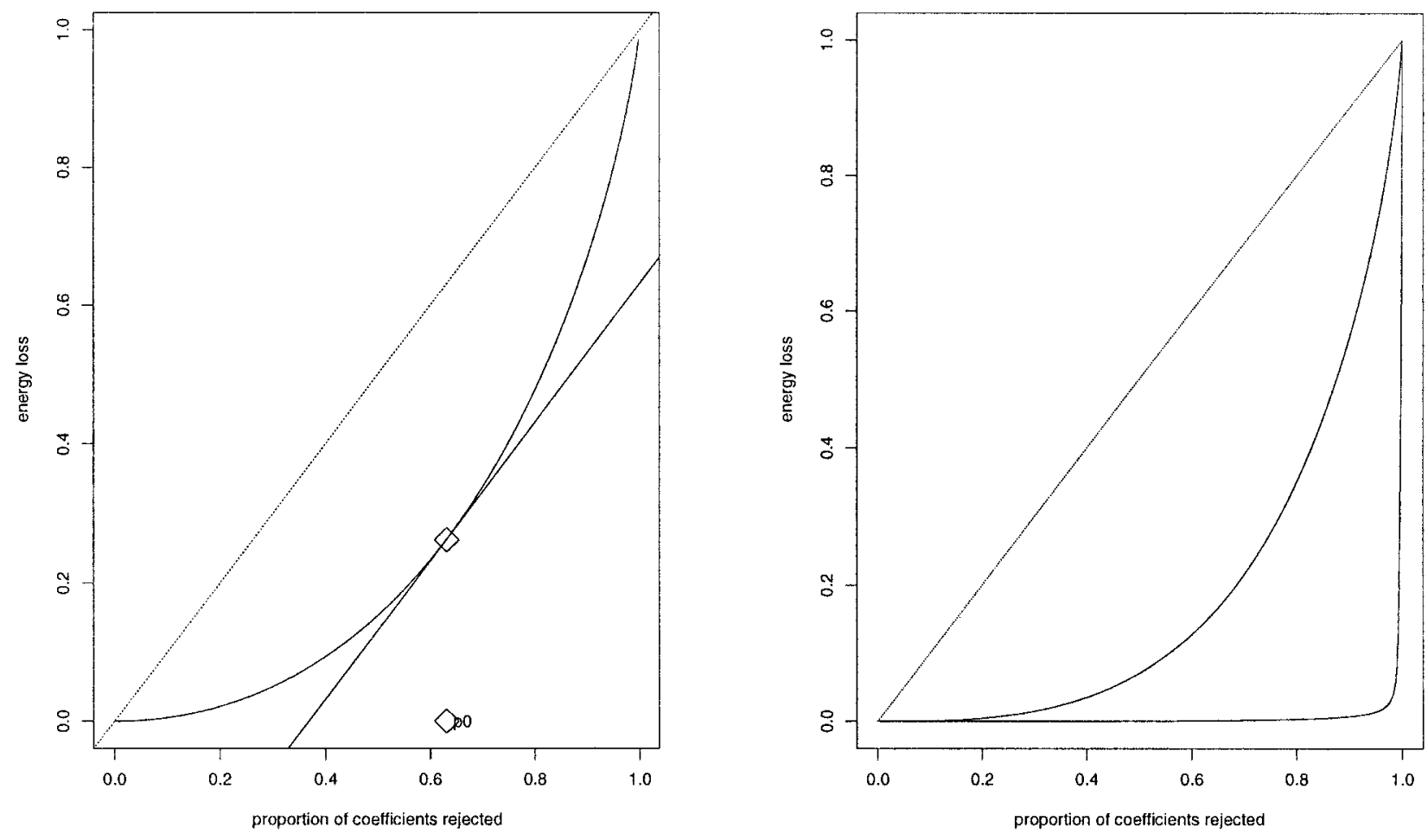

FIG. 1. Left (a): Standard Lorentz-type wavelet thresholding. The diagonal corresponds to a balanced signal and the convex curve corresponds to a disbalanced signal. Notice that the "optimal" proportion $p_{0}$ is determined from the tangent parallel to the diagonal. Right (b): Measured Lorentz curves for longitudinal velocity fluctuations in the time and wavelet domains. The more imbalanced curve corresponds to the Haar wavelet domain.

in degrees of freedom (compression ratios) necessary to quantify energy containing events responsible for heat and momentum fluxes, and (iii) developing low-dimensional models for the turbulent stresses and fluxes as outlined in the review by Lumley et al. (1996), or testing simple phenomenological models such as the attached eddy hypothesis of Townsend (1976).

The second objective is motivated, in part, by the recent results of Wickerhauser et al. (1994), who demonstrated that wavelet packets can achieve compression ratios of enstrophy five times larger than localized cosine transforms for a numerically simulated turbulent vorticity field, while Farge et al. (1992) demonstrated that Fourier transforms can achieve high compression ratios. Two properties are used to explain such high compression ratio: (i) intermittency so that it suffices to retain only a small fraction of time intervals (hereafter, this property is referred to as $P_{i}$ ) or (ii) concentration of energy at low wavenumbers so that it suffices to retain only a few Fourier modes (hereafter, this property is referred to as $P_{e}$ ). To investigate which of the two properties governs compression ratios in ASL turbulence, we consider orthonormal wavelet transforms, linear Fourier rank reduction, and wavelet packets. All these transformations are used to extract the low-dimensional dynamically active components from ASL velocity and temperature time series measurements using energybased conservation measures. A key difference between

TABLE 1. Mean meteorological and turbulence conditions for all runs. The Obukhov length $L$, the friction velocity $u_{*}$, sensible heat flux $\langle w T\rangle$, and the standard deviations of longitudinal and vertical velocities and temperature $\left(\sigma_{u}, \sigma_{w}, \sigma_{T}\right)$ are also shown.

\begin{tabular}{cccccccccc}
\hline \hline Run & $\begin{array}{c}\mathrm{yr} / \mathrm{mo} / \mathrm{d} \\
1995\end{array}$ & $\begin{array}{c}\text { Time } \\
(\text { local })\end{array}$ & $\begin{array}{c}\langle U\rangle \\
\left(\mathrm{m} \mathrm{s}^{-1}\right)\end{array}$ & $\begin{array}{c}\sigma_{u} \\
\left(\mathrm{~m} \mathrm{~s}^{-1}\right)\end{array}$ & $\begin{array}{c}\sigma_{w} \\
\left(\mathrm{~m} \mathrm{~s}^{-1}\right)\end{array}$ & $\begin{array}{c}\sigma_{T} \\
\left({ }^{\circ} \mathrm{C}\right)\end{array}$ & $\begin{array}{c}\langle w T\rangle \\
\left(\mathrm{m} \mathrm{s}^{-1} \mathrm{C}\right)\end{array}$ & $\begin{array}{c}u_{*} \\
\left(\mathrm{~m} \mathrm{~s}^{-1}\right)\end{array}$ & $\begin{array}{c}-L \\
(\mathrm{~m})\end{array}$ \\
\hline 1 & $95 / 7 / 15$ & 0820 & 2.59 & 1.167 & 0.437 & 0.371 & 0.05341 & 0.13441 & 3.5 \\
2 & $95 / 7 / 15$ & 0840 & 2.59 & 0.888 & 0.418 & 0.356 & 0.05429 & 0.14852 & 4.654 \\
3 & $95 / 7 / 15$ & 0920 & 2.63 & 0.762 & 0.439 & 0.426 & 0.07619 & 0.08375 & 0.598 \\
4 & $95 / 7 / 15$ & 0940 & 2.90 & 0.863 & 0.422 & 0.537 & 0.09266 & 0.01778 & 0.005 \\
5 & $95 / 7 / 15$ & 1340 & 2.07 & 0.925 & 0.349 & 0.412 & 0.06578 & 0.17583 & 6.471 \\
6 & $95 / 7 / 16$ & 0940 & 2.23 & 0.795 & 0.308 & 0.533 & 0.08211 & 0.12209 & 1.722 \\
7 & $95 / 7 / 16$ & 1320 & 2.47 & 0.923 & 0.341 & 0.47 & 0.03458 & 0.24602 & 33.729 \\
8 & $95 / 7 / 16$ & 1340 & 2.41 & 0.726 & 0.336 & 0.367 & 0.05176 & 0.18758 & 10.007 \\
\hline
\end{tabular}



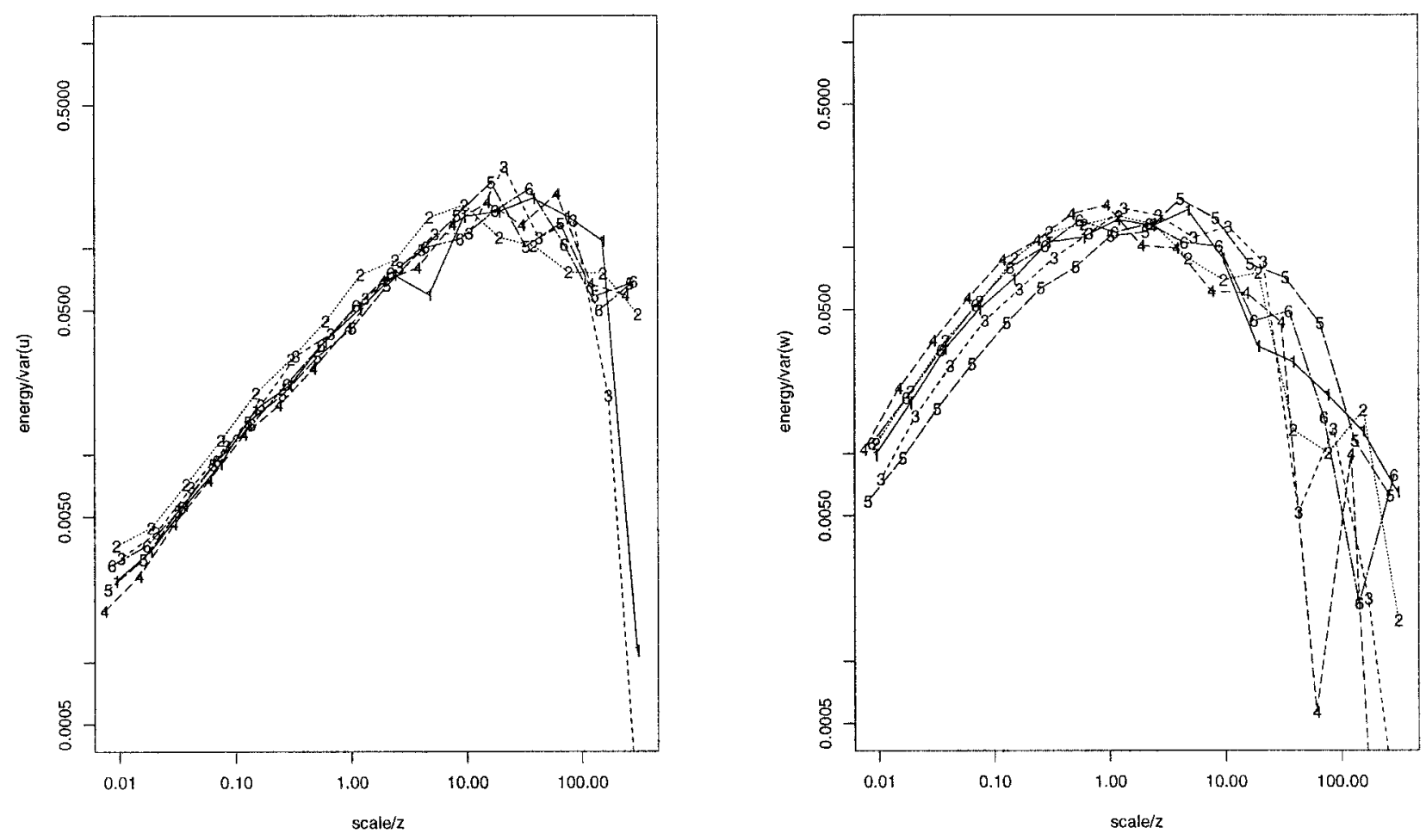

FIG. 2. Total wavelet energy spectra as a function of scale for six runs. The energy is normalized by velocity variances. The scale is normalized by $z$.

wavelet and Fourier methods is in handling the "curse of Heisenberg." Heisenberg's principle states that in modeling time-frequency phenomena, one cannot be precise in both the time and frequency domains. Fourier methods are precise in the frequency domain; however, wavelet transforms adapt the time-frequency precision in a data-dependent manner.

The influence of wavelet bases, thresholding methods, and packet selection criteria on the performance of the above three transformations is evaluated. For this purpose, $56-\mathrm{Hz}$ triaxial sonic anemometer velocity $\left(U_{i}\right)$ and air temperature $\left(T_{a}\right)$ measurements collected in the ASL above a grass-covered forest clearing over a wide range of atmospheric stability conditions are used. Here $U_{i}$ $\left(=U_{1}, U_{2}, U_{3}\right)$ are the longitudinal $(U)$, lateral $(V)$, and vertical $(W)$ velocity; $T_{a}$ is the air temperature; $\left\langle U_{i}\right\rangle$ and $\left\langle T_{a}\right\rangle$ are the time-averaged velocity and temperature, $u_{i}$ and $T$ are the turbulent fluctuations about $\left\langle U_{i}\right\rangle$ and $\left\langle T_{a}\right\rangle$, such that $\left\langle u_{i}\right\rangle=\langle T\rangle=0$; and \langle\rangle is time averaging.

\section{Methods of analysis}

In this section, extraction of low-dimensional flow components using wavelet thresholding methods are considered. In addition, the classic discrete Fourier rank reduction is also reviewed. The basic principles of wavelet transformations can be found in the classic monographs of Daubechies (1992) and Meyer (1992) and will not be considered here. Additional discussion on wave- let applications to signal processing is found in Veterli and Kovačević (1995).

\section{a. Wavelet thresholding models}

It is assumed that a flow variable (e.g., $U_{i}, T_{a}$ ) can be decomposed into a low-dimensional energy-carrying, active part and a high-dimensional passive part that does not contribute to momentum and heat fluxes. This is analogous to the decomposition proposed by Farge et al. (1992) for enstrophy and to Townsend's (1976) attached eddy hypothesis (see Katul and Vidakovic 1996 for a recent review). For the purpose of this study, it is expected that the low-dimensional active part be associated with long-duration eddy motion and can be represented by a function piecewise belonging to the Hölder $^{1}$ smoothness space $\mathcal{H}^{\alpha}, \alpha>1$. As discussed by Meyer

$$
\begin{aligned}
& { }^{1} \text { The Hölder space } \mathcal{H}^{\alpha}(\mathbf{R}) \text { is generalization of spaces "of } n \text { times } \\
& \text { continuously differentiable" functions. It is defined as } \\
& \mathscr{H}^{\alpha}(\mathbf{R})=\left\{f \in L^{\infty}(\mathbf{R}) ; \sup _{x, h} \frac{|f(x+h)-f(x)|}{|h|^{\alpha}}<\infty, 0<\alpha<1\right\} \\
& \mathscr{H}^{\alpha}(\mathbf{R})=\left\{f \in L^{\infty}(\mathbf{R}) \cup C^{n}(\mathbf{R}) ; f^{(n)} \in \mathscr{H}^{\alpha^{\prime}}(\mathbf{R}),\right. \\
& \left.\alpha=n+\alpha^{\prime}, 0<\alpha^{\prime}<1\right\} .
\end{aligned}
$$


TABLE 2. List of wavelet bases used in several turbulence studies. DAUB $\mathrm{n}$ is the notation for standard Daubechies wavelet basis with $n$ vanishing moments.

\begin{tabular}{|c|c|}
\hline Study & Basis \\
\hline Argoul et al. (1989) & Mexican hat \\
\hline Barcy et al. (1991) & Mexican hat and French hat \\
\hline Brunet and Collineau (1994) & Mexican hat \\
\hline Collineau and Brunet (1993) & $\begin{array}{l}\text { Haar, ramp, and jump pseu- } \\
\text { dowavelets }\end{array}$ \\
\hline Everson et al. (1989) & Mexican hat \\
\hline Farge et al. (1992) & Wavelet packets \\
\hline Gao and Li (1993) & Mexican hat \\
\hline Hagelberg and Gamage (1994) & $\begin{array}{l}\text { Symmetric/antisymmetric } \\
\text { cubic spline wavelets }\end{array}$ \\
\hline Hayashi (1994) & Meyer's wavelet \\
\hline Howell and Mahrt (1994b) & $\begin{array}{l}\text { Haar (adaptive multiresolu- } \\
\text { tion filtering) }\end{array}$ \\
\hline Hudgins et al. (1993) & Cubic spline wavelet \\
\hline Hunt et al. (1992) & $\begin{array}{l}\text { Wavelet packets and Fou- } \\
\text { rier transforms }\end{array}$ \\
\hline Katul et al. (1994a,b) & Haar \\
\hline Katul and Parlange (1994) & $\begin{array}{l}\text { Daubechies (1988) com- } \\
\text { pactly supported wavelets }\end{array}$ \\
\hline Katul and Parlange $(1995 a, b)$ & Haar \\
\hline Katul and Vidakovic (1996) & Haar \\
\hline Liandrat and Moret-Bailly (1990) & Mexican hat \\
\hline Lu and Fitzjarald (1994) & Haar \\
\hline Lumley et al. (1996) & $\begin{array}{l}\text { Proper Orthogonal Decom- } \\
\text { position (POD) }\end{array}$ \\
\hline Mahrt (1991) & Haar \\
\hline Mahrt and Howell (1994) & Haar \\
\hline Mayer et al. (1994) & Morlet \\
\hline Meneveau (1991) & $\begin{array}{c}\text { Meyer's wavelet, Haar, } \\
\text { DAUB 2, DAUB } 4\end{array}$ \\
\hline Qiu et al. (1995) & $\begin{array}{l}\text { Pseudowavelet (inverted } \\
\text { ramp) }\end{array}$ \\
\hline Szilagyi et al. (1996) & DAUB 4 \\
\hline Turner and Leclerc (1994) & Haar \\
\hline Turner et al. (1994) & Haar \\
\hline Wickerhauser et al. (1994) & $\begin{array}{l}\text { Wavelet packets and local } \\
\text { cosine transforms }\end{array}$ \\
\hline Yamada and Ohkitani $(1990,1991 a, b)$ & Meyer's wavelet \\
\hline Yee et al. (1996) & Haar \\
\hline Zubair et al. (1992) & $\begin{array}{l}\text { Wavelet packets with Walsh } \\
\text { and Coiflets }\end{array}$ \\
\hline
\end{tabular}

(1992), an important feature of wavelets is that they provide unconditional bases ${ }^{2}$ for a variety of functional spaces (e.g., $\mathbf{L}^{p}, 1<p<\infty$, Hölder spaces and Sobolev spaces). Therefore, given such a representation of longduration eddy motion and given the smoothness properties of wavelet transform, extracting the low-dimensional active part from time series velocity and temperature measurements reduces to a wavelet filtering problem (see appendix A for details). For computational purposes, thresholding wavelet coefficients can be divided into two stages. The first being the choice of the thresholding function $\delta$. Two standard choices are hard and soft thresholding,

\footnotetext{
${ }^{2}$ Informally, a family $\left\{\psi_{i}\right\}$ is an unconditional basis for a space $S$, if one can decide if the element $f=\Sigma_{i} a_{i} \psi_{i}$ belongs to $S$ by looking only at $\left|a_{i}\right| s$.
}

$$
\begin{aligned}
\delta^{\text {hard }}(d, \lambda) & =d \mathbf{1}(|d|>\lambda), \\
\delta^{\text {soft }}(d, \lambda) & =(d-\operatorname{sgn}(d) \lambda) \mathbf{1}(|d|>\lambda),
\end{aligned}
$$

where $\lambda$ is a threshold, $d$ is a wavelet coefficient to which thresholding is applied, and $\mathbf{1}(B)$ is the indicator of condition $B$ taking on the value of 1 when $B$ is satisfied, 0 otherwise.

The second stage is the choice of a threshold criterion. In the following subsections, two global thresholds, well suited for the analysis of turbulence measurements, are described. The choice of global (i.e., the same criterion is applied to all coefficients) thresholds is based on the need to extract the lowest possible dimension necessary to the conservation of energy and fluxes. Scale-adaptive methods, such as SureShrink (Donoho and Johnstone 1994), typically retain a portion of the wavelet coefficients associated with the passive component leading to undesirably large dimensions when compared to global methods (Katul et al. 1994b). A survey of other widely used methods is presented in Nason (1995).

\section{1) DONOHO-JoHnStone UNIVERSAL THRESHOLD}

Donoho and Johnstone (1994) proposed a threshold $\lambda$, based on the following theoretical result concerning the normal probability distribution.

Result

Let $\xi_{i}$ be independent, identically distributed (iid), standard normal random variables. Then

$$
E \max _{i=1, \ldots, n}\left|\xi_{i}\right|=\sqrt{2 \log n}
$$

where $E$ is the expectation operator. This result leads to the following selection of $\lambda$ :

$$
\lambda^{U}=\sqrt{2 \log n} \hat{\sigma}
$$

which Donoho and Johnstone (1994) called universal. The rationale is to remove all wavelet coefficients smaller than the expected maximum of an assumed iid normal noise vector of size $n$.

There are several possibilities for the estimator $\hat{\sigma}$. Almost all methods involve the wavelet coefficients at the finest scale. The signal-to-noise ratio is smallest at high resolutions in a wavelet decomposition for almost all reasonably behaved signals. Hence, standard estimators for $\sigma$ include

$$
\hat{\sigma}=\sqrt{\frac{1}{N / 2-1} \sum_{i=1}^{N / 2}\left(d_{n-1, i}-\bar{d}\right)^{2}}
$$

or a more robust value

$$
\hat{\sigma}=\frac{1}{0.6745} \operatorname{MAD}\left(\left\{d_{n-1, i}, i=1, N / 2\right\}\right),
$$

where MAD is the mean absolute difference and $d_{n-1}$ are the wavelet coefficients at the finest scale. It should be noted that the universal thresholding method does assume that the finest-scale wavelet coefficients are 
TABLE 3. Percent energy and coefficients (in parentheses) retained for selected thresholding methods and basis functions defined as (i) DAUB $n$ : The standard Daubechies wavelet basis (Daubechies 1988, 1992), DAUB 2 is the HAAR basis; (ii) SDAUB $n$ : the least asymmetric Daubechies wavelet basis (Daubechies 1992); (iii) COIF $n$ Coiflets, a wavelet basis in which the scaling function has vanishing moments, in addition to the wavelet function; (iv) BSPL $m \cdot n$ biorthogonal spline bases based on simple polynomial spline functions of degree $m$ and $n$ for $\phi$ and $\psi$ (Chui 1992); (v) VSPL A variation on BSPL designed to achieve near-orthogonality; (vi) PACKD $n$, wavelet packets based on DAUB $n$ filters. The WALSH basis is a wavelet packet based on the HAAR filter.

\begin{tabular}{|c|c|c|c|c|c|c|}
\hline Wavelet & Universal $(U)$ & Lorentz $(U)$ & Universal $(W)$ & Lorentz $(W)$ & Universal $(T)$ & Lorentz $(T)$ \\
\hline FOURIER & & $97.86(2.997)$ & & $96.42(10.27)$ & & $98.06(7.134)$ \\
\hline HAAR & $91.93(0.897)$ & $97.75(2.76)$ & $90.65(3.791)$ & $94.24(6.136)$ & $91.79(1.071)$ & $96.83(3.114)$ \\
\hline DAUB4 & $91.89(0.699)$ & $97.94(2.422)$ & $89.62(2.634)$ & $94.68(5.424)$ & $90.91(0.768)$ & 96.97 (2.799) \\
\hline DAUB6 & $91.92(0.665)$ & $97.98(2.348)$ & $89.13(2.343)$ & $94.65(5.219)$ & $90.58(0.679)$ & $97.08(2.814)$ \\
\hline DAUB8 & $91.62(0.641)$ & $98 \quad(2.39)$ & $88.97(2.273)$ & $94.78(5.198)$ & $90.43(0.652)$ & $97.12(2.762)$ \\
\hline DAUB10 & $91.77(0.647)$ & $98.02(2.352)$ & $88.9 \quad(2.205)$ & $94.84(5.175)$ & $90.36(0.651)$ & $96.97(2.683)$ \\
\hline DAUB12 & $91.73(0.637)$ & $98.01(2.297)$ & $88.6 \quad(2.155)$ & $94.77(5.192)$ & $90.16(0.642)$ & $97.04(2.782)$ \\
\hline DAUB16 & $91.67(0.641)$ & $98.03(2.38)$ & $88.64(2.141)$ & $94.83(5.174)$ & $90.13(0.641)$ & $97.01(2.727)$ \\
\hline DAUB20 & $91.67(0.629)$ & $98 \quad(2.32)$ & $88.43(2.112)$ & $94.7 \quad(5.165)$ & $89.87(0.631)$ & $96.96(2.793)$ \\
\hline SDAUB8 & $91.9 \quad(0.654)$ & $98.03(2.373)$ & $(2.255)$ & $94.73(5.145)$ & $90.65(0.666)$ & $97.12(2.716)$ \\
\hline SDAUB10 & $91.71(0.636)$ & $98.03(2.346)$ & $89.11(2.225)$ & $94.87(5.108)$ & $90.41(0.636)$ & $97.13(2.7)$ \\
\hline SDAUB12 & $91.8 \quad(0.637)$ & $98.02(2.323)$ & $88.82(2.161)$ & $94.78(5.129)$ & $90.53(0.644)$ & $97.09(2.681)$ \\
\hline SDAUB16 & $91.76(0.63)$ & $98.05(2.345)$ & 88.68 (2.09) & $94.78(5.063)$ & $90.44(0.634)$ & $97.1 \quad(2.689)$ \\
\hline SDAUB20 & $91.81(0.629)$ & $98.04(2.34)$ & $88.71(2.126)$ & $94.71(5.029)$ & $90.38(0.627)$ & $97.11(2.668)$ \\
\hline COIF6 & $91.85(0.69)$ & $97.96(2.428)$ & $89.52(2.61)$ & $94.62(5.417)$ & $90.86(0.755)$ & $97.04(2.868)$ \\
\hline COIF12 & $91.86(0.646)$ & $98.01(2.336)$ & $88.9 \quad(2.212)$ & $94.76(5.184)$ & $90.6 \quad(0.653)$ & $97.14(2.754)$ \\
\hline COIF24 & $91.71(0.628)$ & $98.01(2.31)$ & $88.55(2.101)$ & $94.76(5.058)$ & $90.45(0.63)$ & $97.16(2.707)$ \\
\hline BSPL 1.1 & $91.93(0.897)$ & $97.75(2.76)$ & $90.65(3.791)$ & $94.24(6.136)$ & $91.79(1.071)$ & $96.83(3.114)$ \\
\hline BSPL 1.5 & $92.51(0.711)$ & $98.18(2.588)$ & $90.09(2.457)$ & $95.28(5.525)$ & $91.32(0.675)$ & $97.38(2.735)$ \\
\hline VSPL 1 & $91.31(0.554)$ & $97.73(1.897)$ & $88.74(1.935)$ & $94.03(4.151)$ & $90.1 \quad(0.526)$ & $96.69(2.074)$ \\
\hline WALSH & - & $97.69(2.734)$ & - & $94.23(6.133)$ & - & $96.75(3.081)$ \\
\hline PACK D4 & - & $97.85(2.377)$ & - & $94.65(5.369)$ & - & $96.86(2.758)$ \\
\hline PACK D8 & - & $97.90(2.291)$ & - & $94.82(5.116)$ & - & $97.04(2.761)$ \\
\hline PACK D20 & - & $97.94(2.280)$ & - & $94.79(5.054)$ & - & $96.89(2.696)$ \\
\hline
\end{tabular}

Gaussian distributed. However, Katul et al. (1994a,b) demonstrated that ASL turbulence wavelet coefficients at the finest scale are non-Gaussian distributed.

\section{2) LORENTZ THRESHOLDING}

As discussed in Vidakovic (1995) and Katul and Vidakovic (1996), discrete wavelet transformations tend to disbalance the energy for ASL turbulence measurements. Even though the transformations preserve the $l_{2}$ norm of the measurements, the energy of the wavelet transformed time series is concentrated in only few wavelet coefficients. Such a disbalance between the number of wavelet coefficients containing energy and the dimension of the time series offers a practical method to extract low-dimensional energy containing events.

TABLE 4. Compression ratios with Lorentz thresholding for different stability conditions.

\begin{tabular}{cccc}
\hline \hline Run & $u$ & $w$ & $T$ \\
\hline 1 & 99.26 & 94.31 & 97.56 \\
2 & 97.75 & 94.24 & 96.84 \\
3 & 97.38 & 93.72 & 97.31 \\
4 & 97.52 & 98.84 & 97.58 \\
5 & 98.10 & 93.86 & 96.93 \\
6 & 97.82 & 95.99 & 97.89 \\
7 & 98.21 & 93.86 & 98.76 \\
8 & 97.83 & 94.12 & 97.64 \\
\hline
\end{tabular}

A general measure of energy disbalance for a given vector $x$ (in a particular domain such as time, frequency, or wavelet) is the Lorentz curve. The Lorentz curve $L(p)$ is a convex curve describing the cumulative energy (ordinate, $0 \%-100 \%$ ) that is contained in $p \cdot 100 \%$ smallest energy components (abscissa, 0\%-100\%). A sample Lorentz curve is shown in Fig. 1a.

In Fig. 1a, the convexity of the curve relative to the diagonal line (corresponding to the ideally balanced energy) is directly proportional to the energy disbalance. Hence, based on the convexity of the Lorentz curve, a global thresholding criterion is proposed: Threshold (replace by 0 ) $\hat{p}_{0} \cdot 100 \%$ coefficients with smallest energy. The point $\hat{p}_{0}$ is chosen such that the proportion at which the gain (in parsimony) by thresholding an additional wavelet coefficient is smaller than the loss in energy. Both losses are measured on a scale of $0-1$ and are equally weighted as in Fig. 1a. Application of a Lorentz curve to assess energy disbalance in a particular domain is shown in Fig. 1b for longitudinal velocity measurements (see Table 1, run 2 for experimental conditions).

In Fig. 1b, measured Lorentz curves for longitudinal velocities in the time and Haar wavelet domains are displayed. Notice that the Lorentz curve corresponding to the wavelet domain measurements is much more imbalanced when compared to its time domain counterpart; hence, more energy is concentrated in a small number 

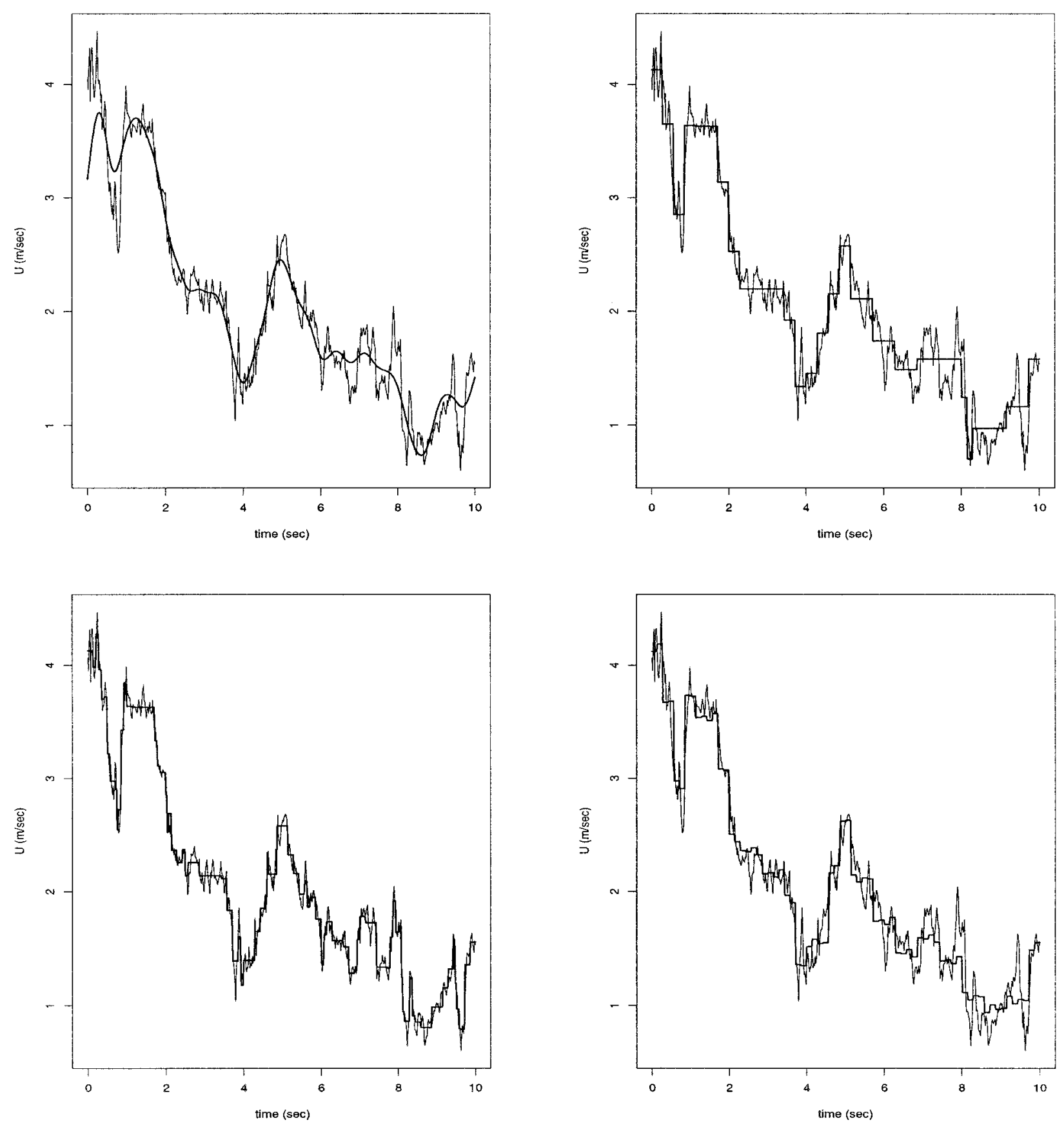

FIG. 3. Comparison between the original and the thresholded longitudinal velocity time series. Upper left (a): Fourier ranking method; Upper right (b): Lorentz thresholding method (using the Haar basis); Lower Left. (c): Universal thresholding method (using the Haar basis); Lower Right (d): Lorentz thresholding method (using the Walsh wavelet-packet basis).

of wavelet coefficients. Differences between energy imbalance in time and wavelet domains, as measured by Lorentz curves, demonstrate that $P_{e}$ is more efficient in isolating the low-dimensional flow components when compared to $P_{i}$.

In Lorentz thresholding, only those coefficients $d_{i}$ for which $d_{i}^{2} \leq \overline{a^{2}}$ are thresholded, where

$$
\bar{d}^{2}=\frac{d_{1}^{2}+d_{2}^{2}+\cdots+d_{n}^{2}}{n}=\frac{\|d\|^{2}}{n} .
$$

It can be shown that the hard Lorentz thresholding is

$$
\delta\left(d_{i}\right)=d_{i} \cdot \mathbf{1}\left(\left|d_{i}\right| \leq \lambda\right),
$$

and by using the orthogonality property of wavelet 
TABLE 5. Comparison of selected thresholding methods for all runs.

\begin{tabular}{|c|c|c|c|c|c|c|}
\hline Variable & Wavelet & Run & Raw & Fourier & Universal & Lorentz \\
\hline \multirow[t]{2}{*}{$\langle u w\rangle\left(\mathrm{m}^{2} \mathrm{~s}^{-2}\right)$} & Haar & $\begin{array}{l}1 \\
2 \\
3 \\
4 \\
5 \\
6 \\
7 \\
8\end{array}$ & $\begin{array}{r}-0.0181 \\
-0.0221 \\
-0.0070 \\
0.0003 \\
-0.0309 \\
-0.0149 \\
-0.0605 \\
-0.0352\end{array}$ & $\begin{array}{r}-0.0177 \\
-0.0214 \\
-0.0072 \\
0.0001 \\
-0.0311 \\
-0.0144 \\
-0.0581 \\
-0.0336\end{array}$ & $\begin{array}{r}-0.0182 \\
-0.0217 \\
-0.0069 \\
0.0004 \\
-0.0312 \\
-0.0149 \\
-0.0602 \\
-0.0347\end{array}$ & $\begin{array}{r}-0.0189 \\
-0.0226 \\
-0.0064 \\
0.0001 \\
-0.0323 \\
-0.0148 \\
-0.0576 \\
-0.0338\end{array}$ \\
\hline & vspline 1 & $\begin{array}{l}1 \\
2 \\
3 \\
4 \\
5 \\
6 \\
6 \\
7 \\
8\end{array}$ & $\begin{array}{r}-0.0181 \\
-0.0221 \\
-0.0070 \\
0.0003 \\
-0.0309 \\
-0.0149 \\
-0.0605 \\
-0.0352\end{array}$ & $\begin{array}{r}-0.0177 \\
-0.0214 \\
-0.0072 \\
0.0001 \\
-0.0311 \\
-0.0144 \\
-0.0581 \\
-0.0336\end{array}$ & $\begin{array}{r}-0.0181 \\
-0.0219 \\
-0.0068 \\
0.0003 \\
-0.0309 \\
-0.0150 \\
-0.0603 \\
-0.0349\end{array}$ & $\begin{array}{r}-0.0158 \\
-0.0238 \\
-0.0068 \\
0.0005 \\
-0.0321 \\
-0.0157 \\
-0.0590 \\
-0.0342\end{array}$ \\
\hline \multirow[t]{2}{*}{$\left\langle w T_{a}\right\rangle\left(\mathrm{m} \mathrm{s}^{-1}{ }^{\circ} \mathrm{C}\right)$} & Haar & $\begin{array}{l}1 \\
2 \\
3 \\
4 \\
5 \\
6 \\
6 \\
7 \\
8\end{array}$ & $\begin{array}{l}0.0534 \\
0.0543 \\
0.0762 \\
0.0927 \\
0.0658 \\
0.0821 \\
0.0346 \\
0.0518\end{array}$ & $\begin{array}{l}0.0481 \\
0.0497 \\
0.0696 \\
0.0880 \\
0.0627 \\
0.0798 \\
0.0303 \\
0.0478\end{array}$ & $\begin{array}{l}0.0524 \\
0.0530 \\
0.0747 \\
0.0918 \\
0.0652 \\
0.0817 \\
0.0335 \\
0.0505\end{array}$ & $\begin{array}{l}0.0502 \\
0.0509 \\
0.0721 \\
0.0899 \\
0.0640 \\
0.0810 \\
0.0320 \\
0.0491\end{array}$ \\
\hline & vspline & $\begin{array}{l}1 \\
2 \\
3 \\
4 \\
5 \\
6 \\
7 \\
8\end{array}$ & $\begin{array}{l}0.0534 \\
0.0543 \\
0.0762 \\
0.0927 \\
0.0658 \\
0.0821 \\
0.0346 \\
0.0518\end{array}$ & $\begin{array}{l}0.0481 \\
0.0497 \\
0.0696 \\
0.0880 \\
0.0627 \\
0.0798 \\
0.0303 \\
0.0478\end{array}$ & $\begin{array}{l}0.0530 \\
0.0537 \\
0.0755 \\
0.0923 \\
0.0655 \\
0.0819 \\
0.0339 \\
0.0510\end{array}$ & $\begin{array}{l}0.0515 \\
0.0520 \\
0.0737 \\
0.0906 \\
0.0647 \\
0.0816 \\
0.0327 \\
0.0498\end{array}$ \\
\hline
\end{tabular}

transformations, $\lambda$ can be expressed as $\|\mathbf{x}\| / \sqrt{n}$, where $\mathbf{x}=\left\{x_{1}, \cdots, x_{n}\right\}$ is the measured flow variable and $\|\mathbf{x}\|$ is the standard $l_{2}$ norm of $\mathbf{x}$.

In contrast to universal thresholding, (4) does not assume any probabilistic structure for the wavelet coefficients. This method differs from the adaptive multiresolution algorithm proposed by Howell and Mahrt (1994a,b) since no window width or upper cutoff length are required. Furthermore, this method is well suited for wavelet packet thresholding, as discussed in appendix B.

\section{b. Fourier thresholding}

The one-dimensional, de-meaned time series $\mathbf{x}=\left\{x_{i}\right.$, $i=1, \cdots, N\}$ is transformed to frequency domain using

$$
\mathbf{f}=\frac{1}{N} \operatorname{FFT}(\mathbf{x})
$$

where FFT is the Fourier transform of $\mathbf{x}$ and $\mathbf{x}$ is, as before, some turbulent flow variable.

The Fourier transformation $\mathbf{f}$ of the time series $\mathbf{x}$ is a complex-valued sequence. Thresholding is performed by setting all entries $f_{i}$ for which $\left|f_{i}\right|<\lambda$ to 0 . In order to compare with other wavelet thresholding methods, the threshold $\lambda$ is set to be a Lorentz threshold $(=\|x\| / \sqrt{n})$. The thresholded sequence $\mathbf{f}^{*}$ has a complex-valued inverse Fourier transformation. Hence, as an estimator of the thresholded time series,

$$
\mathbf{x}^{a}=\left|\operatorname{IFFT}\left(\mathbf{f}^{*}\right)\right|
$$

is used, where IFFT is the inverse Fourier transform.

This method is, in essence, hard thresholding of Fourier coefficients using a Lorentz threshold function. Due to the nonlocality of the Fourier kernel, soft thresholding is not recommended. Furthermore, since a comparison between Fourier ranking and wavelet approaches is considered, hard thresholding [Eq. (1)] is used throughout for consistency.

\section{Experimental setup}

The velocity and air temperature measurements were carried out on 12-16 July 1995 at $5.2 \mathrm{~m}$ above the ground surface over an Alta Fescue grass site at the Blackwood division of the Duke Forest in Durham, North Carolina. During this time period, a heat wave resided in North Carolina for several days after it swept from the Midwest to the East Coast. During the experiment, maximum mean air temperature up to $38^{\circ} \mathrm{C}$ was measured in Durham. The sky condition during these 5 days was clear with low to moderate winds. The site is 

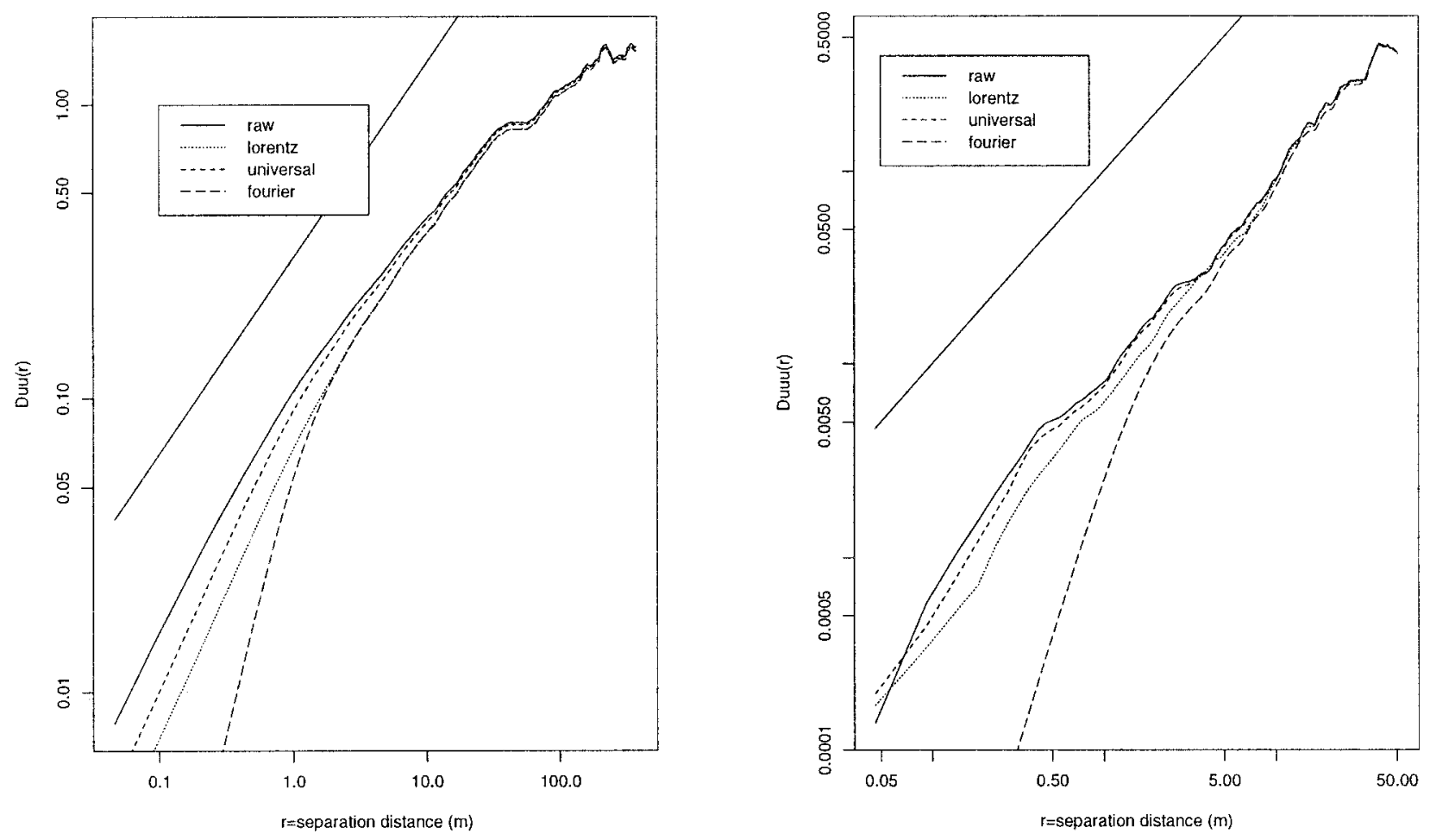

FIG. 4. Comparison between original and thresholded longitudinal velocity structure functions. Left (a): Second-order structure function $D_{\text {ии }}$; Right (b): third-order structure function $D_{\text {ии }}$. The solid lines are from the original time series. The power laws are from K41.

a $480 \mathrm{~m}$ by $305 \mathrm{~m}$ grass-covered forest clearing $\left(36^{\circ} 2^{\prime} \mathrm{N}\right.$, $79^{\circ} 8^{\prime} \mathrm{W}$, elevation $=163 \mathrm{~m}$ ), and a mast situated at 250 and $160 \mathrm{~m}$ from the north-end and west-end portions of a $12 \mathrm{~m}$ tall Loblolly pine forest edge, respectively, was used to mount a triaxial sonic anemometer (Szilagyi et al. 1996). The three velocity components $\left(U_{1}, U_{2}, U_{3}\right)$ and air temperature were measured using a triaxial ultrasonic anemometer (Gill Instruments/1012R2). Sonic anemometers measure the velocity by sensing the effect of wind on the transit times of sound pulses traveling in opposite directions across a known instrument path distance $d_{s l}(=0.149 \mathrm{~m}$ in this study). A key disadvantage of sonic anemometers is the wavenumber $(K)$ distortion due to averaging along the finite sonic path $d_{s l}$. This distortion is limited to separation distances $r<d_{s l}$ or $K>d_{s l}^{-1}$ (Wyngaard 1981). In this experiment, we focus on eight runs that span a wide range of meteorological, atmospheric stability, and turbulence conditions presented in Table 1.

The sampling frequency $\left(f_{s}\right)$ and period $\left(T_{p}\right)$ were 56 $\mathrm{Hz}$ and $19.5 \mathrm{~min}$, resulting in $N=65536$ measurements per velocity component per run. The $56-\mathrm{Hz}$ sampling frequency is the maximum achievable frequency by the Gill sonic anemometer and is used in this experiment. The sampling period is sufficiently long to resolve the energy containing scales of the $u$ time series, as shown in Fig. 2. In Fig. 2 Haar wavelet total energy as a function of scale is depicted for $u$ (left panel) and $w$ (right panel). Notice that energetic scales, comparable to the atmospheric boundary layer height, are resolved in the $u$ time series. For the $w$ time series, the energy is concentrated in scales comparable to $z$. Further details about the experimental setup can be found in (Szilagyi et al. 1996).

\section{Results and discussion}

As discussed in Farge (1992), the wavelet basis function can inject properties in the transformed series that are dependent on the wavelet choice rather than the process under consideration. Currently, the choice of the wavelet basis function in the analysis of turbulence measurements is arbitrary (see Table 2 for summary of some studies). This variability in analyzing wavelets motivated us to consider two elements in the wavelet thresholding process: the wavelet basis and the thresholding criterion. Table 3 presents the influence of the wavelet basis function and the thresholding approach on a sample run (run 2). The library of orthonormal wavelets used in Table 3 extends from compactly supported Daubechies wavelets used by Katul and Vidakovic (1996) and Katul et al. (1994a,b) to infinitely supported spline wavelets used by Yamada and Ohkitani (1990) and Meneveau (1991a,b). Notice in Table 3 that the energy conservation and compression ratios are robust to the choice of wavelet bases but not to the wavelet thresholding method. It should be noted in Table 3 that the Universal method resulted in lower 


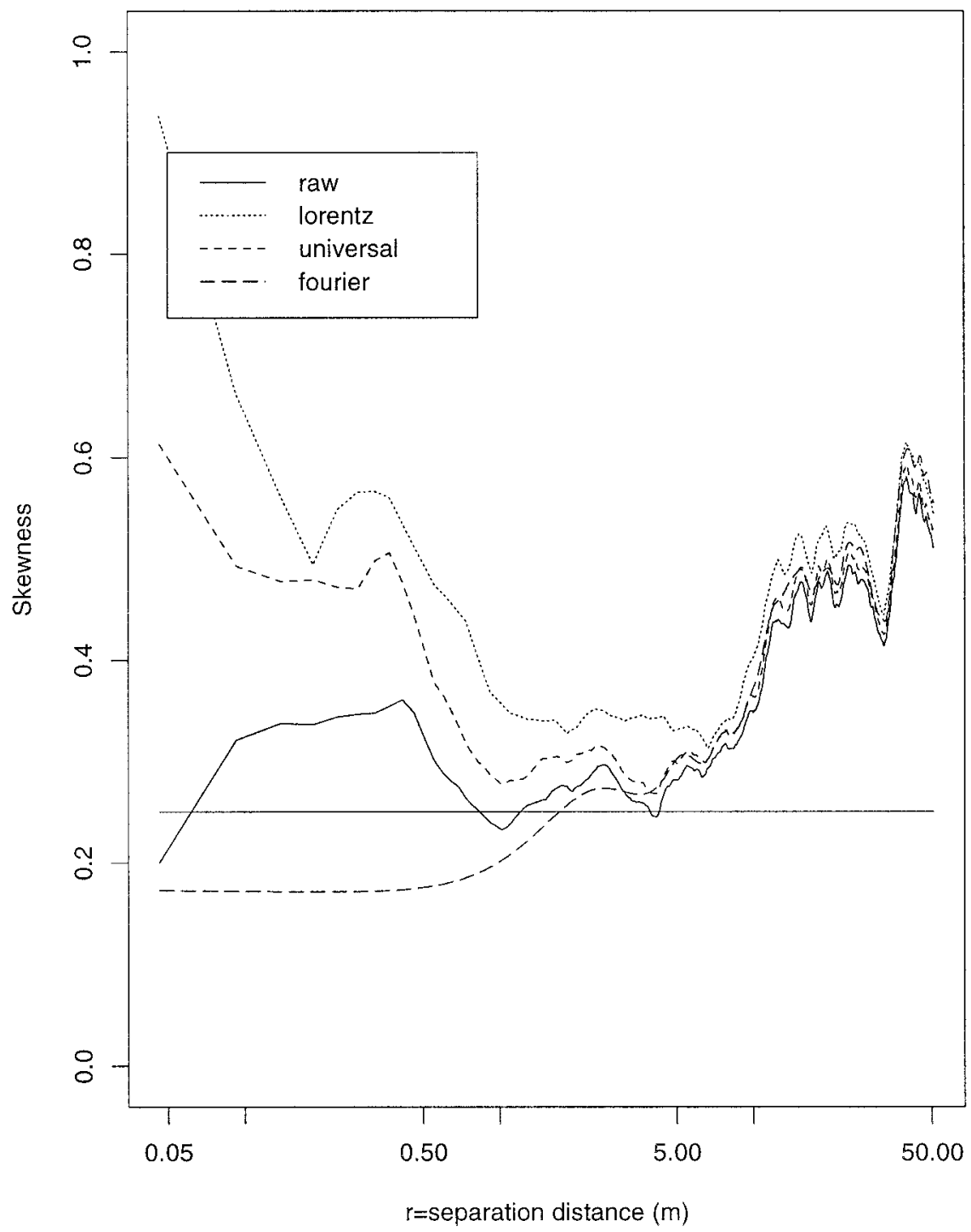

FIG. 5. Comparison between original and thresholded longitudinal velocity structure skewness. The solid line is from the original time series. The constant skewness is determined from local isotropy and the Kolmogorov constant.

energy loss at the expense of lower compression ratios when compared to Lorentz threshold functions for all atmospheric stability conditions. However, the energy losses due to the Lorentz threshold are well within measurement variance uncertainty (e.g., Lumley and Panofsky 1964). Since our objective is to concentrate energy in the least number of coefficients, Lorentz thresholding is recommended. Furthermore, since Universal thresholding assumes a Gaussian probabilistic structure at the fine scales, it may not be valid for velocity and temperature measurements as discussed in Katul et al. (1994a,b).

In order to investigate the variability in wavelet compression ratios with atmospheric stability conditions, the analysis in Table 3 was repeated for all runs using the Haar wavelet and Lorentz thresholding. We found that the performance of Lorentz thresholding is robust to changes in $z / L$ (unstable conditions only) as evidenced by Table 4 , and the values are consistent with the bare soil measurements described in Katul and Vidakovic (1996).

From Table 4 it appears that the compression ratios for $u$ and $T$ are higher than $w$. The results from Table 4 and Fig. 2 suggest that, for flow variables for which energy is concentrated at scales much larger than $z$, the wavelet compression ratios (Lorentz thresholding) are expected to be higher than for flow variables for which energy is concentrated at intermediate scales (comparable to $z$ ). This difference in compression performance is attributed to the fact that intermediate scales require more wavelet coefficients than larger scales in wavelet decompositions. 


\section{a. Validation}

In order to test whether wavelet thresholding conserves the dynamical properties of large- and smallscale eddy motions, two approaches are used. The first approach evaluates whether the small number of wavelet coefficients $(3 \%-10 \%)$ are sufficient to reproduce the heat and momentum fluxes for each thresholding model. The second approach evaluates whether thresholding is restricted to wavelet coefficients associated with smallscale eddy motion as described by K41 power laws. These two approaches are considered next.

\section{b. Heat and momentum flux conservation}

While Table 3 demonstrates that all thresholding models are able to concentrate much of the turbulent energy in a few coefficients, little is known whether these limited coefficients can reproduce covariances between turbulent variables. That is, the thresholding methodology extracts low-dimensional organized perturbations $\left(U^{(0)}\right.$, $\left.W^{(o)}, T_{a}^{(o)}\right)$ from velocity $(U, W)$ and temperature $\left(T_{a}\right)$ time series measurements, given by

$$
\begin{aligned}
U & =U^{(o)}+U^{(r)} \\
W & =W^{(o)}+W^{(r)} \\
T_{a} & =T_{a}^{(o)}+T_{a}^{(r)},
\end{aligned}
$$

and filters out the high-dimensional part $\left(U^{(r)}, W^{(r)}\right.$, $\left.T_{a}^{(r)}\right)$. As a graphical illustration, Fig. 3 compares the original $(U)$ and the thresholded $\left(U^{(o)}\right)$ longitudinal velocity time series for all thresholding methods.

What remains to be tested is whether

$$
\begin{aligned}
\langle u w\rangle & =\left\langle u^{(o)} w^{(o)}\right\rangle \\
\left\langle w T_{a}\right\rangle & =\left\langle w^{(o)} T_{a}^{(o)}\right\rangle
\end{aligned}
$$

is valid as in Townsend's (1976) attached eddy hypothesis; that is, whether the limited coefficients from each time series are sufficient to conserve the turbulent fluxes. For this purpose, the momentum and heat fluxes using the measured $(N=65536)$, Fourier, Universal, and Lorentz thresholded time series are compared for all eight runs in Table 5. The Haar and v-spline bases are used as illustration since they represent the extremes in energy conservation and percent coefficients thresholded, as in Table 3. The calculations in Table 5 are performed by thresholding and reconstructing each time series using the nonzero Fourier, wavelet, or wavelet packet coefficients. From the reconstructed longitudinal, vertical, and temperature time series, the momentum and sensible heat fluxes are computed and compared to the original fluxes computed from the 65536 measurements per run. All thresholding methods in Table 5 reproduced the measured fluxes (momentum and heat) to within 5\%. While both wavelet Lorentz filtering and Fourier ranking marginally underestimated the measured covariances, the underestimation appears larger in the Fourier approach despite near equality in the number of coef- ficients thresholded. However, this is a minor underestimation for both methods given the uncertainty in the measured covariances for ASL flows (see, e.g., run 4). Table 5 also suggests that the compression results are insensitive to the choice of wavelet basis.

\section{c. Small-scale filtering and K41 power laws}

In K41, the second-order $\left(D_{\text {uи }}\right)$ and third-order $\left(D_{\text {uиu }}\right)$ structure functions are given by

$$
\begin{aligned}
D_{\text {ии }} & =C_{2}\langle\epsilon\rangle^{2 / 3} r^{2 / 3} \\
D_{\text {иии }} & =-\frac{4}{5}\langle\epsilon\rangle r,
\end{aligned}
$$

where $r$ is the separation distance, $C_{2}(=0.55)$ is the Kolmogorov constant, and $\langle\epsilon\rangle$ is the mean turbulent kinetic energy dissipation rate (Monin and Yaglom 1975) per unit mass. In Eq. (7), intermittency corrections to the dissipation rate, in the sense of Kolmogorov (1962), are not considered. These corrections are minor for second-order structure functions and absent for third-order structure functions. This type of intermittency is discussed elsewhere (Frisch 1995; Katul et al. 1994b).

In theory, $\mathrm{K} 41$ is strictly valid for $r / z \ll 1$, but, in practice, the above power laws have been observed for $r>z$, especially for longitudinal velocity measurements in the ASL. In Figs. $4 \mathrm{a}$ and $4 \mathrm{~b}, D_{u и}$ and $D_{\text {uиu }}$ are shown for the original $(N=65536)$ and thresholded time series for all thresholding models. Also, the structure skewness, given by

$$
S(r)=\frac{D_{\text {uии }}}{D_{\text {uи }}^{3 / 2},}
$$

is shown in Fig. 5. Taylor's (1938) frozen turbulence hypothesis is used to convert the time to space domain. Run 2 is chosen because the measured squared turbulent intensity does not exceed 0.1 , and hence distortions due to Taylor's hypothesis are minimal (Wyngaard and Clifford 1977). The structure function analysis in Figs. 4 and 5 is restricted to $r>d s l(=0.15 \mathrm{~m})$; however, the full range of structure function measurements are shown. Notice that the influence of thresholding is apparent for $r \leq z / 2$ in all methods. The thresholding distorts the fine scales (which follow K41 power laws) but not the large scales $(r>z / 2)$ responsible for much of the variances and covariances. This analysis further supports the hypothesis that the high-dimensional component is associated with detached eddies.

\section{Conclusions}

This study demonstrated the following:

1) Wavelet thresholding is capable of isolating organized events responsible for much of the variances and covariances, irrespective of the wavelet basis. Hence, a key advantage to utilizing such wavelet thresholding methods to extract low-dimensional eddy mo- 
tion from time series measurements is their robustness with respect to the analyzing wavelet function.

2) Haar wavelet bases in conjunction with Lorentz filtering achieve compression ratios and variance-covariance conservations comparable to more sophisticated wavelet packet methods used by Zubair et al. (1992), Farge et al. (1992), and Wickerhauser et al. (1994).

3) Thresholding in the Fourier domain using a Lorentz thresholding function can achieve compression ratios comparable to orthonormal wavelet and waveletpacket techniques. This, in part, is attributed to the significant disbalance in Lorentz curves for the energies of Fourier (frequency localization) and Haar wavelet (adaptive time-frequency localization) coefficients. By comparing Lorentz curves in the time and frequency domains for all flow variables, we found that the frequency Lorentz curves are substantially more imbalanced than their time domain counterparts. Hence, this analysis demonstrates that while turbulence is intermittent in the time domain with a power-law decay in energy, the latter is the main contributor to high compression ratios.

4) The ability of wavelet thresholding to retain the low-dimensional dynamically active events and filter out the high-dimensional dynamically passive part was tested via structure function approaches in relation to K41. It was shown that these low-dimensional events are not associated with the fine scales but with scales much larger than $z / 2$.

Finally, concentrating the turbulent activity in limited number of wavelet coefficients does suggest that turbulence can be decomposed into a low-dimensional component responsible for much of the turbulent energy production and transport dynamics superimposed on a high-dimensional passive or inertial component. Therefore, this study further supports Townsend's (1976) attached eddy hypothesis and Morrison's et al. (1992) laboratory results.

Acknowledgments. The authors would like to thank Cheng-I Hsieh for his assistance during the experiment at the Duke Forest. This project was funded, in part, by the National Science Foundation Grant DMS-9626159 and the U.S. Department of Energy (DOE) through the National Institute for Global Environmental Change (NIGEC), the Southeast Regional Center at the University of Alabama, Tuscaloosa (DOE Cooperative Agreement DE-FC03-90ER61010). We thank Dr. Christopher Bretherton and the three anonymous referees for their helpful comments.

\section{APPENDIX A}

\section{Linkage between Filtering and Thresholding}

In this appendix, linkage between wavelet filtering and thresholding is discussed. Based on Meyer's theorem (Meyer 1992):
Let $\phi$ be a fixed scaling function corresponding to the wavelet $\psi$. Let $\phi_{j k}(x)=2^{j / 2} \phi\left(2^{j} x-k\right)$ and $\psi_{j k}(x)=$ $2^{j / 2} \psi\left(2^{j} x-k\right)$.

$$
\begin{aligned}
\text { Hence, } f \text { is } \mathcal{H}^{\alpha} \text { if } \\
\left.\qquad f, \phi_{0 k}\right\rangle \mid \leq C, \forall k \in \mathbf{Z} ; \\
\left|\left\langle f, \psi_{j k}\right\rangle\right| \leq C 2^{-j(\alpha+1 / 2)}, \forall j \geq 0, \forall k \in \mathbf{Z},
\end{aligned}
$$

where $\left\langle f, \phi_{j k}\right\rangle$ and $\left\langle f, \psi_{j k}\right\rangle$ represent the inner products defining the wavelet coefficients. The linkage between smoothness of the function and the energy of its wavelet coefficients is established. Hence, the terms filtering and thresholding are used interchangeably.

\section{APPENDIX B}

\section{Overview of Wavelet Packets}

In this appendix, an overview of wavelet packet thresholding, as utilized in Table 3, is presented. Wavelet packets, introduced by Coifman and Meyer (1991), represent natural generalization of wavelet orthonormal bases. Due to their extreme adaptability, they are considered an indispensable tool in acoustic signal processing and are gaining attention in turbulence research (Zubair et al. 1990; Farge et al. 1992; Hunt et al. 1992; Wickerhauser et al. 1994).

For completeness, a brief overview of wavelet packets definitions is given.

Let $\mathbf{h}$ and $\mathbf{g}$ be low-pass and high-pass filters, respectively, forming a pair of quadrature mirror filters. The following sequence of functions,

$$
\begin{gathered}
W_{2 n}(x)=\sum_{k} h_{k} \sqrt{2} W_{n}(2 x-k) \\
W_{2 n+1}(x)=\sum_{k} g_{k} \sqrt{2} W_{n}(2 x-k) \\
n=0,1,2, \cdots,
\end{gathered}
$$

represents a library from which orthonormal bases can be selected. In the above, increasing the parameter $n$ amounts to increasing the number of oscillations in $W_{n}$.

In standard notation, $W_{0}(x)=\phi(x), W_{1}(x)=\psi(x)$, where $\phi$ and $\psi$ are the scaling and wavelet functions, respectively, generated by filters $\mathbf{h}$ and $\mathbf{g}$. For $h_{0}=h_{1}$ $=1 / \sqrt{2}$; the system $W_{n}(x)$ is the well-known Walsh basis.

The library of all wavelet packets is overcomplete and any arbitrary selection of functions from it may not form an orthonormal or complete set. The following theorem gives a constructive approach to select an orthonormal wavelet packet basis via appropriate selection of the scale $(j)$ and the oscillation $(n)$ indices in the functions defined by

$$
\begin{aligned}
& W_{j, n, k}=2^{-j / 2} W_{n}\left(2^{-j} x-k\right), \\
& \quad j \in \mathbf{Z}, \quad n \in \mathbf{N}, \quad k \in \mathbf{Z},
\end{aligned}
$$

where $k$ is an arbitrary translation index. 


\section{Theorem}

Let the collection $\{j, n\}$ be such that the dyadic intervals $I_{j, n}=\left[2^{j} n, 2^{j}(n+1)\right]$ form a disjoint covering of the interval $[0, \infty)$. Then $\left\{W_{j, n, k}, j \in \mathbf{Z}, n \in \mathbf{N}, k \in\right.$ $\mathbf{Z}$ \} is a complete orthonormal basis for $\mathbf{L}_{2}$.

With a basis defined as in the above theorem, any $\mathbf{L}_{2}$ function $f(x)$ can be decomposed as

$$
f(x)=\sum_{j} \sum_{n} \sum_{k} d_{j n k} W_{j n k}(x) .
$$

Fast algorithms for calculating all possible $d_{j n k}$ for discrete signals and selecting the best basis with respect to the entropy cost criterion can be found in Wickerhauser (1994). The thresholding of wavelet packet coefficients in Table 3 was performed using the Lorentz thresholding function. Due to the ambiguity in the definition of a signal to noise ratio for packets, universal thresholding was not applied.

\section{REFERENCES}

Argoul, F., A. Arneodo, G. Grasseau, Y. Gagne, E. J. Hopfinger, and U. Frisch, 1989: Wavelet analysis of turbulence reveals the multifractal nature of the Richardson cascade. Nature, 338, 51-53.

Barcy, E., A. Arneodo, U. Frisch, Y. Gagne, and E. Hopfinger, 1991: Wavelet analysis of fully developed turbulence data and measurement of scaling exponents. Turbulence and Coherent Structures, O. Metais and M. Lesieur, Eds., Kluwer Academic, 203215.

Brunet, Y., and S. Collineau, 1994: Wavelet analysis of diurnal and nocturnal turbulence above a maize crop. Wavelets in Geophysics, E. Foufoula-Georgiou and P. Kumar, Eds., Academic Press, 129-150.

Chui, C. K., 1992: An Introduction to Wavelets. Academic Press, 264 $\mathrm{pp}$.

Coifman, R. R., and Y. Meyer, 1991: Remarques sur l'analyse de Fourier à fenêtre. C. R. Acad. Sci. Paris Sér. I, Math., 312, 259261.

Collineau, S., and Y. Brunet, 1993: Detection of turbulent coherent motion in a forest canopy: Wavelet analysis. Bound.-Layer Meteor., 65, 357-379.

Daubechies, I., 1988: Orthonormal bases of compactly supported wavelets. Commun. Pure Appl. Math., XLI, 909-996.

- 1992: Ten Lectures on Wavelets. Vol. 61, CBMS-NSF Regional Conference Series in Applied Mathematics, SIAM, 357 pp.

Donoho, D., and I. Johnstone, 1994: Ideal spatial adaptation by wavelet shrinkage. Biometrika, 81, 425-455.

Everson, R., L. Sirovich, and K. R. Sreenivasan, 1990: Wavelet analysis of the turbulent jet. Phys. Lett. A, 145, 314-322.

Farge, M., 1992: Wavelet transforms and their applications to turbulence. Annu. Rev. Fluid Mech., 24, 395-457.

— E. E. Goirand, Y. Meyer, F. Pascal, and M. V. Wickerhauser, 1992: Improved predictability of two-dimensional turbulent flows using wavelet packet compression. Fluid Dyn. Res., 10, 229-250.

Frisch, U., 1995: Turbulence: The Legacy of A. N. Kolmogorov. Cambridge University Press, $296 \mathrm{pp}$.

Gamage, N., and C. Hagelberg, 1993: Detection and analysis of microfronts and associated coherent events using localized transforms. J. Atmos. Sci., 50, 750-756.

Gao, W., and B. L. Li, 1993: Wavelet analysis of coherent structures at the atmosphere-forest interface. J. Appl. Meteor., 32, 17171725.

Hagelberg, C. R., and N. K. Gamage, 1994: Application of structure preserving wavelet decompositions to intermittent turbulence: A case study. Wavelets in Geophysics, E. Foufoula-Georgiou and P. Kumar, Eds., Academic Press, 45-80.
Hayashi, T., 1994: An analysis of wind velocity fluctuations in the atmospheric surface layer using an orthonormal wavelet transform. Bound.-Layer Meteor., 70, 307-326.

Howell, J. F., and L. Mahrt, 1994a: An adaptive decomposition: Application to turbulence. Wavelets in Geophysics, E. FoufoulaGeorgiou and P. Kumar, Eds., Academic Press, 107-128.

$\longrightarrow$, and — 1994b: An adaptive multiresolution data filter: Applications to turbulence and climatic time series. J. Atmos. Sci., 51, 2165-2178.

Hudgins, L. H., M. E. Mayer, and C. A. Friehe, 1993: Fourier and wavelet analysis of atmospheric turbulence. Progress in Wavelet Analysis and Applications, Y. Meyer and S. Roques, Eds., Frontieres, 491-498.

Hunt, J. C. R., N. K. R. Kevlahan, J. C. Vassilicos, and M. Farge, 1992: Wavelets, fractals, and fourier transforms: Detection and analysis of structure. Wavelets, Fractals, and Fourier Transforms: New Development and Applications, M. Fargre, J. C. R. Hunt, and J. C. Vassilicos, Eds., Oxford Press, 1-38.

Katul, G. G., and M. B. Parlange, 1994: On the active role of temperature in surface-layer turbulence. J. Atmos. Sci., 51, 21812195.

_ , and _ 1995a: The spatial structure of turbulence at production wavenumbers using orthonormal wavelets. Bound.-Layer Meteor., 75, 81-108.

$\longrightarrow$, and $\longrightarrow$ 1995b: Analysis of land-surface heat fluxes using the orthonormal wavelet approach. Water Resour. Res., 31, 2743-2749.

— tached eddy motion in the atmospheric surface layer using Lorentz wavelets. Bound.-Layer Meteor., 77, 153-172.

— J. D. Albertson, C. R. Chu, and M. B. Parlange, 1994a: Intermittency in atmospheric surface layer turbulence: The orthonormal wavelet representation. Wavelets in Geophysics, E. Foufoula-Georgiou and P. Kumar, Eds., Academic Press, 81-105.

-, M. B. Parlange, and C. R. Chu, 1994b: Intermittency, local isotropy, and non-Gaussian statistics in atmospheric surface layer turbulence. Phys. Fluids, 6, 2480-2492.

Kolmogorov, A. N., 1941: The local structure of turbulence in incompressible viscous fluid for very large Reynolds numbers. Dokl. Akad. Nauk. SSSR, 30, 301-305.

— 1962: Refinement of previous hypotheses concerning the local structure of turbulence in a viscous incompressible fluid at high Reynolds number. J. Fluid Mech., 13, 82-85.

Liandrat, J., and F. Moret-Bailly, 1990: The wavelet transform: Some applications to fluid mechanics and turbulence. Eur. J. Mech., B/Fluids, 9, 1-9.

Lu, C. H., and D. R. Fitzjarald, 1994: Seasonal and diurnal variations of coherent structures over a deciduous forest. Bound.-Layer Meteor., 69, 43-69.

Lumley, J., and H. Panofsky, 1964: The Structure of Atmospheric Turbulence. John Wiley and Sons, $239 \mathrm{pp}$.

— G. Berkooz, J. Elezgary, P. Holmes, A. Poje, and C. Volte, 1996: Fundamental aspects of incompressible and compressible flows. Simulation and Modeling of Turbulent Flows, ICASE/ LaRC Series in Computational Science and Engineering, Oxford, $5-78$.

Mahrt, L., 1991: Eddy asymmetry in the sheared heated boundary layer. J. Atmos. Sci., 48, 472-492.

_ , and F. Howell, 1994: The influence of coherent structures and microfronts on scaling laws using global and local transforms. J. Fluid Mech., 260, 247-270.

Mayer, M. E., L. Hudgins, and C. A. Friehe, 1994: Wavelet spectra of buoyant atmospheric turbulence. Wavelets: Theory, Algorithms, and Applications, C. Chui, L. Montefusco, and L. Puccio, Eds., Academic Press, 533-541.

Meneveau, C., 1991a: Dual spectra and mixed energy cascade of turbulence in the wavelet representation. Phys. Rev. Lett., 66, $1450-1452$.

- $1991 \mathrm{~b}$ : Analysis of turbulence in the orthonormal wavelet representation. J. Fluid Mech., 232, 469-520. 
Meyer, Y., 1992: Wavelets and Operators. Cambridge Studies in Advanced Mathematics, Vol. 37, Cambridge University Press, 223 pp.

Monin, A. S., and A. M. Yaglom, 1975: Statistical Fluid Mechanics. Vol. II. J. Lumley, Ed., The MIT Press, 879 pp.

Morrison, J. F., C. S. Subramanian, and P. Bradshaw, 1992: Bursts and the law of the wall in turbulent boundary layers. J. Fluid Mech., 241, 75-108.

Nason, G., 1995: Choice of the threshold parameter in wavelet function estimation. Wavelets in Statistics, A. Antoniadis and G Oppenheim, Eds., Lecture Notes in Statistics, Springer-Verlag, 281-299.

Qiu, J., K. T. Paw U, and R. H. Shaw, 1995: Pseudo-wavelet analysis of turbulent patterns in three vegetation layers. Bound.-Layer Meteor. 72, 177-204.

Raupach, M., R, Antonia, and S. Rajagopalan, 1991: Rough-wall turbulent boundary layers. Appl. Mech. Rev. 44, 1-25.

Szilagyi, J., G. G. Katul, M. B. Parlange, J. D. Albertson, and A. T. Cabill, 1996: The local effect of intermittency on the inertial subrange energy spectrum of the atmospheric surface layer. Bound.-Layer Meteor., 79, 35-50.

Taylor, G. I., 1938: The spectrum of turbulence. Proc. Roy. Soc. London, A, 164, 476-490.

Tewfik, A., D. Sinha, and P. Jorgensen, 1992: On the optimal choice of a wavelet for signal representation. IEEE Trans. Inf. Theory, 38, 747-765.

Townsend, A., 1976: The Structure of Turbulent Shear Flow. Cambridge University Press, 429 pp.

Turner, B. J., and M. Y. Leclerc, 1994: Conditional sampling of coherent structures in atmospheric turbulence using wavelet transform. J. Atmos. Oceanic Technol., 11, 205-209.

_, — M. Gauthier, K. E. Moore, and D. R. Fitzjarald, 1994:
Identification of turbulence structures above a forest canopy using a wavelet transform. J. Geophys. Res., 99, 1919-1926.

Veterli, M., and Kovačević, J., 1995: Wavelets and Subband Coding. Prentice Hall, 488 pp.

Vidakovic, B., 1995: Unbalancing data with wavelet transformations. Wavelet Applications in Signal and Image Processing III, Proc. SPIE 2569, Vol 2, 845-857.

Wickerhauser, M. V., 1994: Adapted Wavelet Analysis from Theory to Software. A. K. Peters, 486 pp.

- M. Farge, E. Goirand, E. Wesfreid, and E. Cubillo, 1994: Efficiency comparison of wavelet packet and adapted local cosine bases for compression of a two-dimensional turbulent flow. Wavelets: Theory, Algorithms, and Applications, C. Chui, L. Montefusco, and L. Puccio, Eds., Academic Press, 509-531.

Wyngaard, J., 1981: Cup, propeller, vane, and sonic anemometer in turbulence research. Annu. Rev. Fluid Mech., 13, 399-427.

— turbulence spectra. J. Atmos. Sci., 34, 922-929.

Yamada, M., and K. Ohkitani, 1990: Orthonormal expansion and its application to turbulence. Prog. Theor. Phys.: Prog. Lett., 86, 819-823.

, and - 1991a: Orthonormal wavelet analysis of turbulence. Fluid Dyn. Res., 8, 101-115.

, and - 1991b: An identification of energy cascade in turbulence by orthonormal wavelet analysis. Prog. Theor. Phys., 86, 799-815.

Yee, E., R. Chan, P. R. Kosteniuk, C. A. Biltoft, and J. F. Bowers, 1996: Multiscaling properties of concentration fluctuations in dispersing plumes revealed using an orthonormal wavelet decomposition. Bound.-Layer Meteor., 77, 173-207.

Zubair, L., K. R. Sreenivasan, and M. V. Wickerhauser, 1992: Turbulent signals and images using wavelet-packets. Studies in Turbulence, T. Gatski, S. Sarkar, and C. Speziale, Eds., SpringerVerlag, $602 \mathrm{pp}$ 